\title{
Randomised comparison of spinal anaesthesia with bupivacaine or levobupivacaine for hip surgery in elderly subjects.
}

Valle. A; Fernandez. D; Gordo. F; Pontes. C; Vives. R; Oliva. JC; Izquierdo. A; Calo. N; Garcia. C; Hernandez. Ch; Colilles. C; Anesthesiology department $H$. Parc Taulí from Sabadell, España.

\section{BACKGROUND:}

Bupivacaine and levobupivacaine have similar pharmacokinetic and pharmacodynamic characteristics, and are used regularly in spinal anaesthesia. Whether potential differences in their haemodynamic and anaesthetic profiles could determine differential risk of complications in elderly subjects is controversial.

\section{OBJECTIVES:}

To compare intrathecally administered levobupivacaine versus racemic bupivacaine through regional cerebral $\mathrm{O} 2$ saturation, anaesthetic parameters, postoperative cognitive status and neurological complications.

\section{METHODS:}

The trial included 58 patients aged 70 or older undergoing surgery for hip fracture and requiring spinal anaesthesia, who were randomized to receive bupivacaine or levobupivacaine. The primary outcome was the proportion of intraoperative time with regional cerebral desaturation ( $\geq 20 \%$ reduction from baseline), as monitored by near -infrared spectroscopy. Secondary end points included hemodynamic parameters, level of sensitive and motor block, changes in Short Portable Mental Status Questionnaire, and neurological complications ( bradypsychia, disartria, encephalopathy or symptoms of confusional)

\section{RESULTS:}

\section{Proportion of intraoperative time with regional cerebral desaturation ( $>20 \%$ reduction from baseline)}

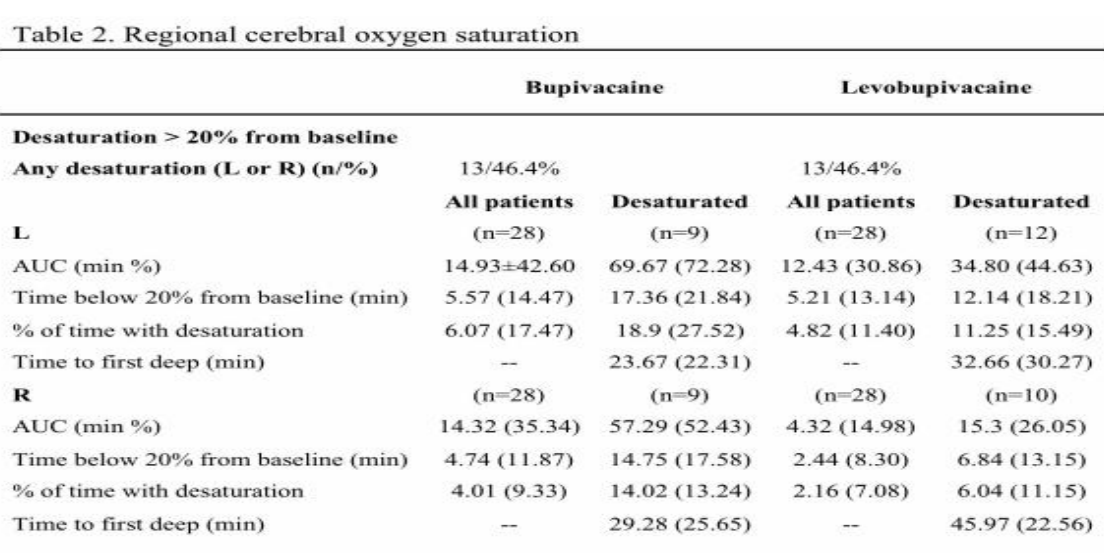

-Thirty-seven patients $(66 \%)$ had some type of regional cerebral desaturation

-The proportion of intraoperative time with desaturation were similar in both groups for both hemispheres.

- Intraoperative desaturation was found to be associated with lower $\mathrm{R}$ baseline $\mathrm{rCSO} 2$ and lower minimum MAP during the anaesthetic procedure.

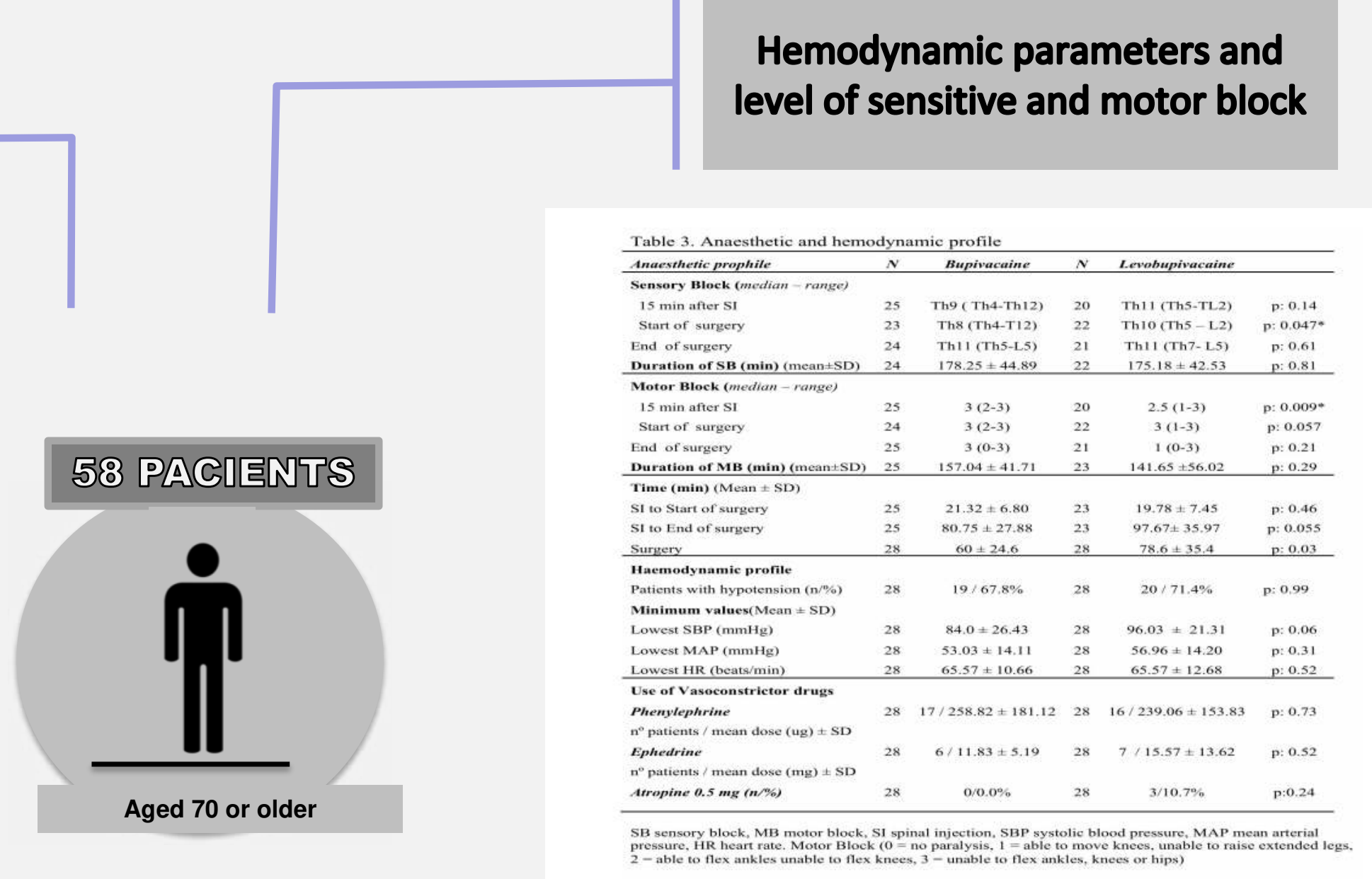

Hemodynamic parameters and level of sensitive and motor block
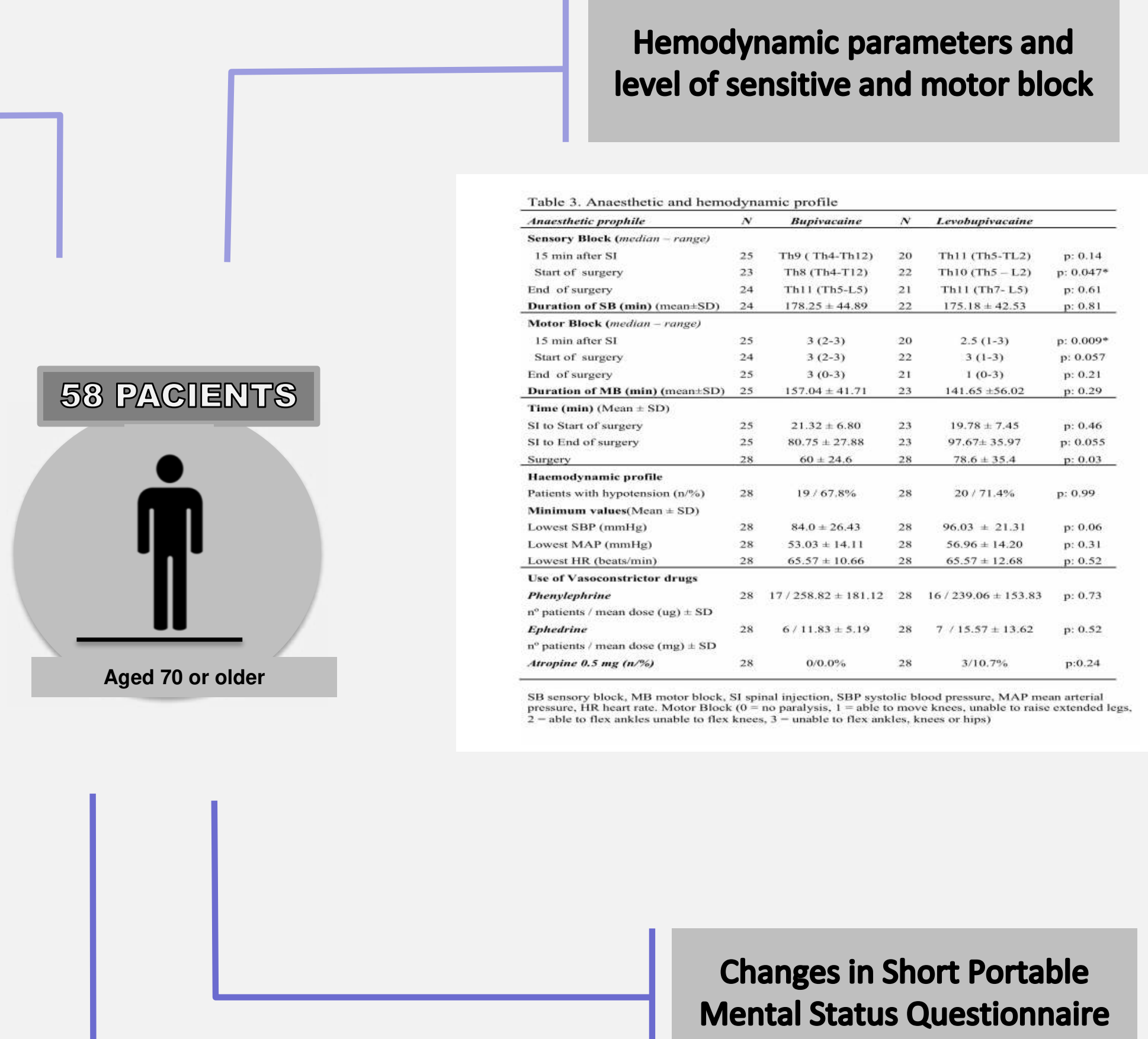

Changes in Short Portable Mental Status Questionnaire

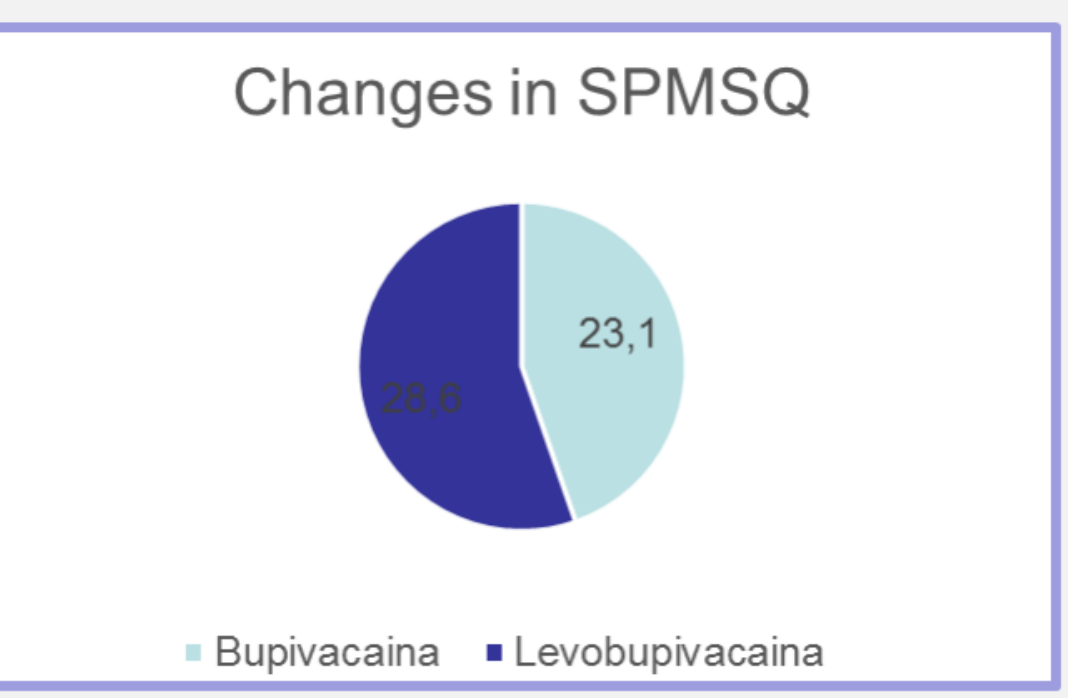

\section{CONCLUSIONS:}

- Bupivacaine and levobupivacaine differed in sensitive and motor block levels at the start of surgery, but not in hemodynamic parameters.

- Neurological complications were more frequent with bupivacaine.

- Avoiding low intraoperatory mean blood pressure is important to preserve cerebral oxygenation, specially in subjects with low regional oxygenation of right hemisphere. 